\title{
Synthesis of Palladium Nanocubes/Nanorods and Their Catalytic Activity for Heck Reaction of Iodobenzene
}

\author{
Hao Ding, Jiling Dong ${ }^{1, *}$ \\ School of Chemistry and Chemical Engineering, Chongqing University of Science and Technology, Chongqing 401331, China \\ ${ }^{1}$ School of Metallurgy and Materials Engineering, Chongqing University of Science and Technology, Chongqing 401331, China
}

*Correspondence to:

Dong J,

Tel: $+86-23-65023278$

Fax: +86-23-65023278

E-mail: dongjiling@cqust.edu.cn

Received December 30, 2015

Revised April 13, 2016

Accepted April 13, 2016
Palladium has been used as a catalyst not only in Suzuki and Heck cross coupling reaction in organic chemistry, but also in automobile industry for the reduction of vehicle exhausts. The catalytic activity of Pd nanoparticles depends strongly on their size and exposed crystalline facets. In this study, the single crystalline palladium nanocubes/nanorods were prepared in the presence of polyvinyl pyrrolidone (PVP) and potassium bromide $(\mathrm{KBr})$ using the polyol method. Selected area diffraction pattern and high-resolution transmission electron microscopy (TEM) were performed by TEM. The result shows that the ratio of $\mathrm{KBr} / \mathrm{PVP}$ is the key factor to determine whether the product is cubes or rods. The as-prepared Pd nanocubes were highly uniform in both size and shape. The ordered packing structures including monolayer and multilayer can be fabricated via the ratecontrolled evaporation of solution solvent. The catalytic activity of these Pd nanocubes towards heck reaction of iodobenzene with acrylate or acrylic acid was found to be higher than that of Pd nanorods. We suspect it is caused by the difference of energy state while Pd nanocubes is $\{100\}$ plane and nanorods is $\{111\}$ plane.

Key Words: Palladium nanocubes/nanorods, Catalytic activity, Heck reaction of iodobenzene

\section{INTRODUCTION}

Noble-metal catalysts are widely used in industry because of their high activity and selectivity for a large number of important chemical reactions. Generally, Palladium has been used as a catalyst not only in Suzuki and Heck cross coupling reaction in organic chemistry (Burda et al., 2005; Kim et al., 2002), but also in automobile industry for the reduction of vehicle exhausts (Nishihata et al., 2002). It has been recognized that the size of Pd particles is one of the most important factors that dictate the performance of a catalyst, recent investigation showed that the catalytic activity of Pd particles depends strongly on the exposed crystalline facets (Shen et al., 2003; Xiao et al., 2009). For example, the Pd (100) deposited onto Au or Pt electrode showed an improved catalytic activity over Pt catalyst for formic acid oxidation (Baldauf \& Kolb, 1996). This is probably because the oxidation rate of formic acid on Pd (100) is higher than that on Pt (100). To discover the catalytic activity and the structure-activity relationship of $\mathrm{Pd}$ nano particles in reactions, various methods have been devoted to the preparation of Pd nanocatalysts, such as oleylamine-mediated synthesis (Mazumder \& Sun, 2009), metal chloride reduction process (Larsen et al., 2006; Xu et al., 2007), amonia-mediated polyol process (Li et al., 2007), alcohol reduction process (Wang et al., 2008; Xiong et al., 2005, 2007) and thermal decomposition of palladium complexes (Kim et al., 2003). Therefore, the study on new strategies for shape control on Pd NPs is quite desirable for the discovery of Pd nanocatalysts. In this paper, we report the synthesis of $\mathrm{Pd}$ nanocubes/nanorods using a facile polyol reduction process. The as-prepared $\mathrm{Pd}$ nanocubes/nanorods have an fcc crystalline structure. The catalytic activity of these Pd nanocubes towards Heck reaction was found higher than that of as-prepared Pd nanorods. It was consistent with the

(a) This is an open-access article distributed under the terms of the Creative Commons Attribution Non-Commercial License (http://creativecommons.org/licenses/by-nc/4.0) which permits unrestricted noncommercial use, distribution, and reproduction in any medium, provided the original work is properly cited.

Copyrights @ 2016 by Korean Society of Microscopy 
fact found in Pd single crystalline electrode that Pd (100) surface is highly catalytic-active (Hoshi et al., 2006).

\section{MATERIALS AND METHODS}

The Pd nanocubes/nanorods were synthesized by a modified polyol method (Xiong et al., 2007). In a $50 \mathrm{~mL}$ flask, polyvinyl pyrrolidone (PVP; molecular weight $=58,000)$ and potassium bromide $(\mathrm{KBr})$ were dissolved in ethylene glycol (EG), and then heated in air under magnetic stirring at certain temperature for 1 hour. The palladium precursor was prepared in another vessel by dissolving $\mathrm{Na}_{2} \mathrm{PdCl}_{4}$ in a mixed solution of water and EG. This solution was injected into the flask using a syringe pump. The reaction mixture was then heated up to $150^{\circ} \mathrm{C}$ in air and kept at this temperature for 1 hour. The product was collected by centrifugation and washed with acetone and then with the mixture of ethanolcyclohexane (1:5 in volume ratio) for five times to remove the excess $\mathrm{EG}, \mathrm{NaBr}$, and PVP.

$\mathrm{X}$-ray diffractometer (XRD) pattern was recorded with $\mathrm{CuK}_{\alpha}$ radiation source. Transmission electron microscopy (TEM) and selected area diffraction pattern (SADP) were performed.
TEM samples were prepared by placing one drop of the Pd nanocubes or nanorods solution on a carboncoated copper grid. The catalytic activity for Heck reaction of Iodobenzene was also investigated of both Pd nanorods and nanocubes.

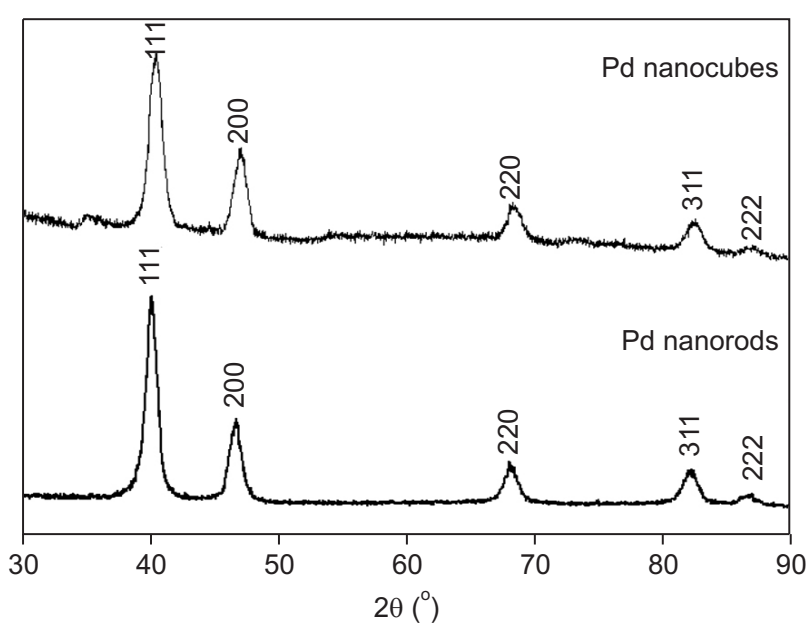

Fig. 1. X-ray diffractometer pattern of Pd nanorods and nanocubes.
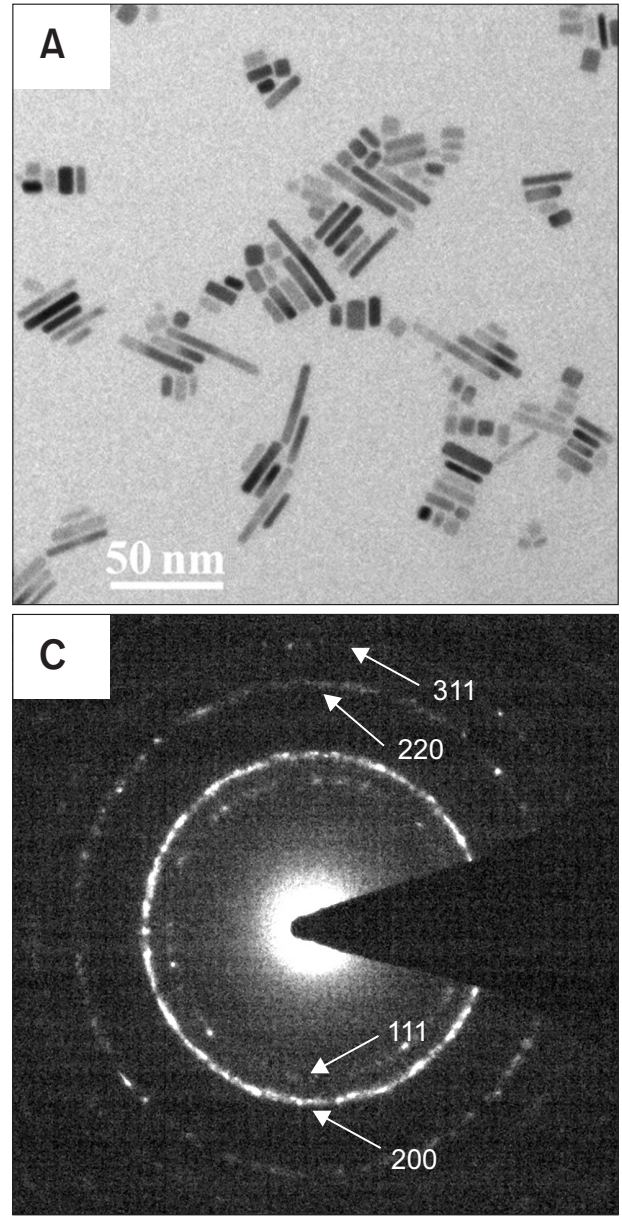
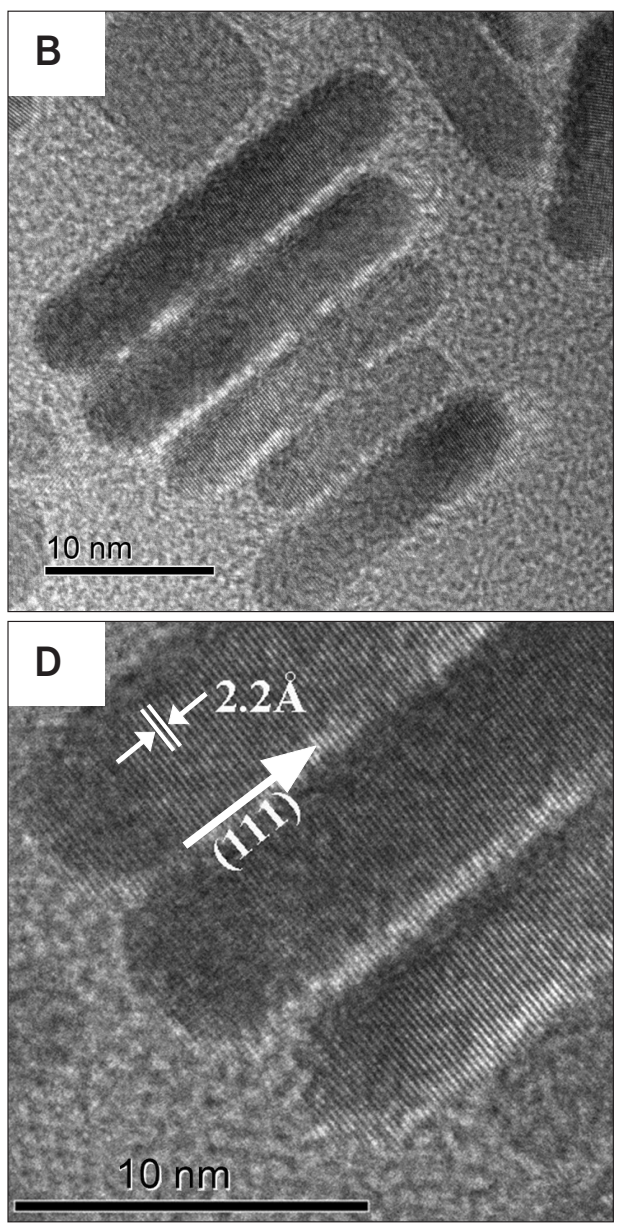

Fig. 2. Transmission electron microscopy (TEM) images of Pd nanorods. (A) Low magnification image. (B) High magnification image of a monolayer. (C) Selected area diffraction pattern. (D) High-resolution TEM image. 


\section{RESULTS AND DISCUSSION}

Pd nanorods and nanocubes were synthesized in the presence of PVP and $\mathrm{KBr}$ using polyol method with different ratio of KBr/PVP (Pd:Br:PVP=1:1:1 to 1:5:5), inject speed $(0.33 \mathrm{~mL} /$ $\mathrm{min}$ to $1 \mathrm{~mL} / \mathrm{min})$ and temperature $\left(100^{\circ} \mathrm{C}\right.$ to $\left.150^{\circ} \mathrm{C}\right)$. As the result, with excessive bromide and PVP, inject slowly and low temperature, it tends to form Pd nanorods. Whether with less bromide and PVP, inject fast and high temperature, Pd nanocubes forms easily.

The crystal phase of Pd nanorods and nanocubes were characterized by XRD as shown in Fig. 1. The reflection peaks of both samples match well with the fcc crystalline structure of bulk Pd (Joint Committee on Powder Diffraction Standards [JCPDS], card No. 46-1043), corresponding to the $\{111\}$, $\{200\},\{220\},\{311\}$, and $\{222\}$ planes of Pd. The intensity ratio of (111) to (200) peak in Pd nanocubes decreases slightly as compared to the standard value calculated from JCPDS data (from 2.38 to 1.97), indicating that the reflection of $\{100\}$ planes dominates in Pd nanocubes, meanwhile, the intensity ratio of (111) to (200) peak in Pd nanorods is similar to the standard value.
Fig. 2A and B show the typical TEM images of as-prepared $\mathrm{Pd}$ nanorods. The close view of a monolayer of $\mathrm{Pd}$ nanocubes (Fig. 1B) indicates that the Pd nanorods are in well-defined shape. SADP image of Pd nanorods clearly shows typical fcc structure with diffraction rings of $\{111\},\{200\},\{220\}$, and $\{311\}$, indicating high crystallinity (Fig. 1C). The highresolution TEM (HRTEM) image of Pd nanorods (Fig. 1D) shows lattice fringes with an interfringe distance of approximately $2.2 \AA$, which is close to the interplane distance of the $\{111\}$ planes in the fcc structured Pd. It demonstrates that the surfaces of fcc-structured Pd nanocubes are selectively exposed $\{111\}$ plane. The HRTEM image also confirms that the nanorods are of single phase Pd. Meanwhile, TEM images of Pd nanocubes are showed in Fig. 3, with an interfringe distance of approximately $1.9 \AA$ at surface, which is close to the interplane distance of the $\{200\}$ planes in the fcc structured Pd.

$\mathrm{KBr}$ plays a critical role in the formation of nanorods or nanocubes because the $\mathrm{Br}^{-}$ions from $\mathrm{KBr}$ act as an etchant to affect the growth of Pd nanorods and nanocubes. Introduction of the bromide results in chemisorption on the surface of Pd seeds and alters the order of surface free
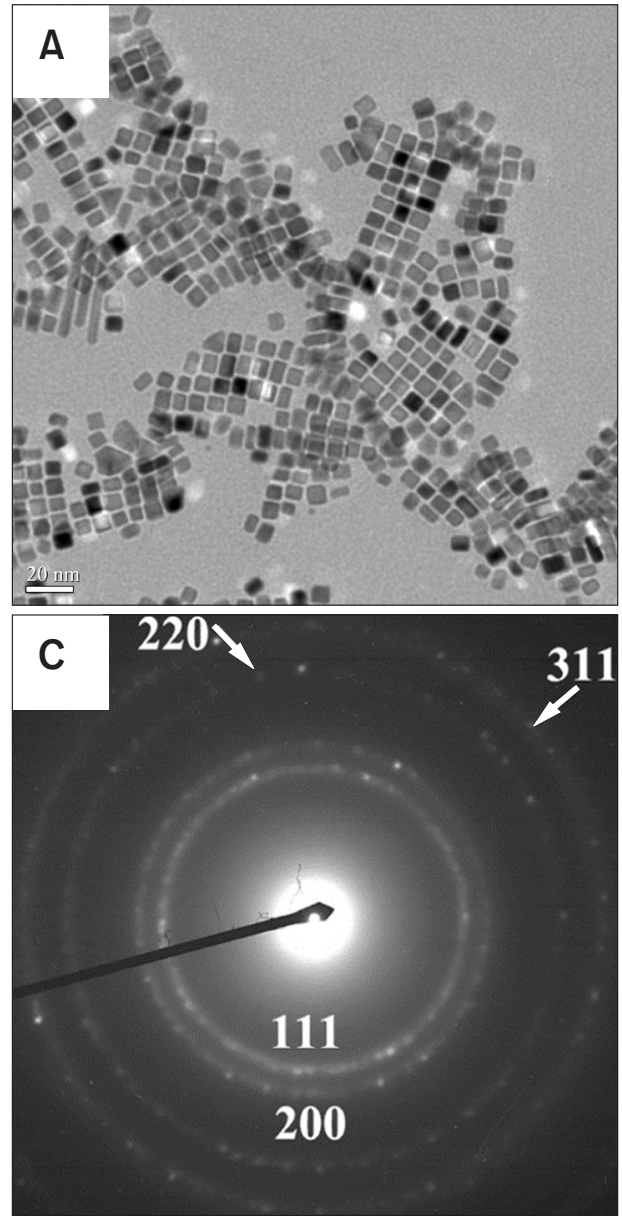
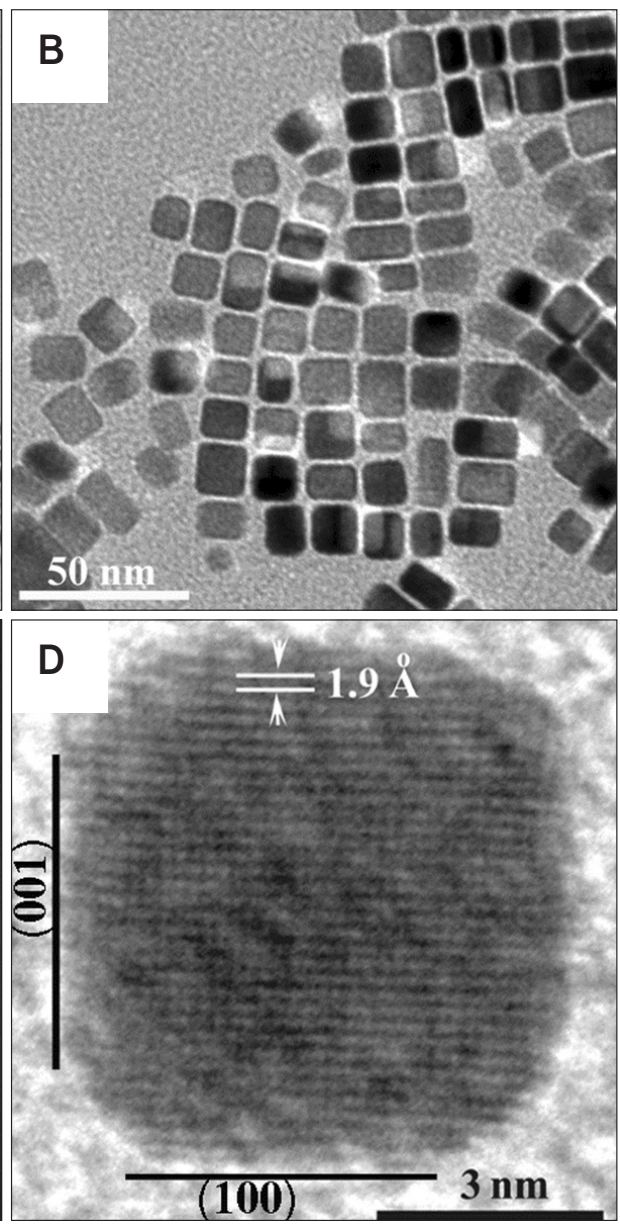

Fig. 3. Transmission electron microscopy (TEM) images of Pd nanocubes. (A) Low magnification image. (B) High magnification image of a monolayer. (C) Selected area diffraction pattern. (D) High-resolution TEM image. 


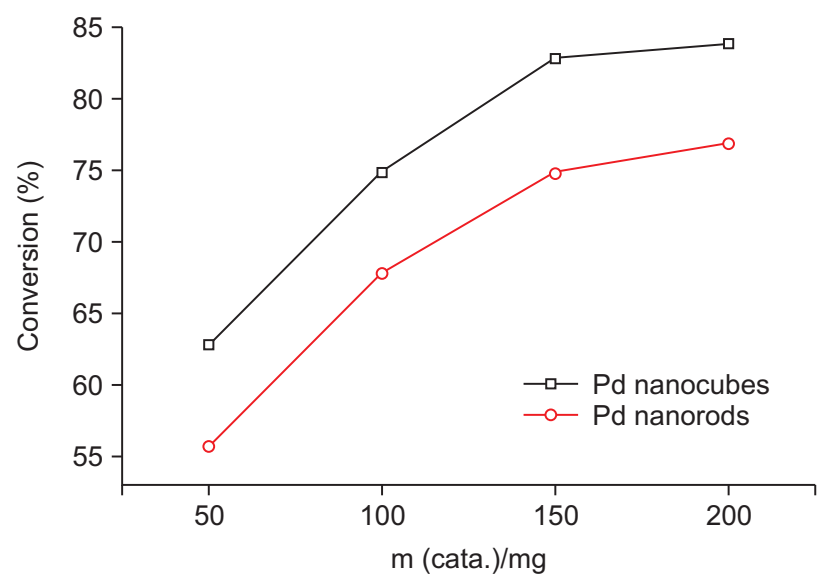

Fig. 4. Catalytic performance of Pd nanorods/nanocubes catalyst for Heck reaction of iodobenzene with acrylic acid.

energies. In reaction process, the addition of Pd atoms from solution deposited on to the Pd nanocrystal surface can be etched by $\mathrm{Br}^{-}$ions, which leads to the anisotropic growth of Pd nanostructures and promotes the formation of $\{200\}$ planes. The existence of specific anions has been proven to be an effective etchant for the control synthesis of metal nanostructures. We found that only irregular polyhedral particles can be obtained with PVP alone. On the other hand, we can only obtain aggregate Pd nanoparticles when using $\mathrm{KBr}$ and no PVP.

To study the catalytic activity of these Pd nanocubes/ nanorods towards Heck reaction, as-prepared Pd nanocubes/ nanorods were used as catalyst in the reaction of iodobenzene and methyl acrylate or acrylic acid, the equation is as follow:<smiles>CCCCCCCCCOC(=O)C=C[I-]c1ccccc1</smiles>

The conversion of reactant was analyzed by gas chromatograph. Fig. 4 shows the Heck reaction of iodobenzene and acrylic acid, the product, base and solvent was cinnamic acid, tributylamine, N, N-Dimethylformamide (DMF), respectively, with the temperature of $100^{\circ} \mathrm{C}$ and the reaction time of 1 hour. Fig. 5 shows the Heck reaction of iodobenzene and acrylic acid methyl ester, the product was cinnamic acid methyl ester, and the solvent changed to N-Methyl pyrrolidone (NMP) with same other conditions as above. As seen in this series of reactions, the quantity of nanocubes/ nanorods increased from $50 \mathrm{mg}$ to $200 \mathrm{mg}$ while keeping the ratio of iodobenzene to acrylic acid is 1:1.5. We found that the conversion was increased by quantity of catalyst, but the degree of increasing decreased; also the conversion of Pd

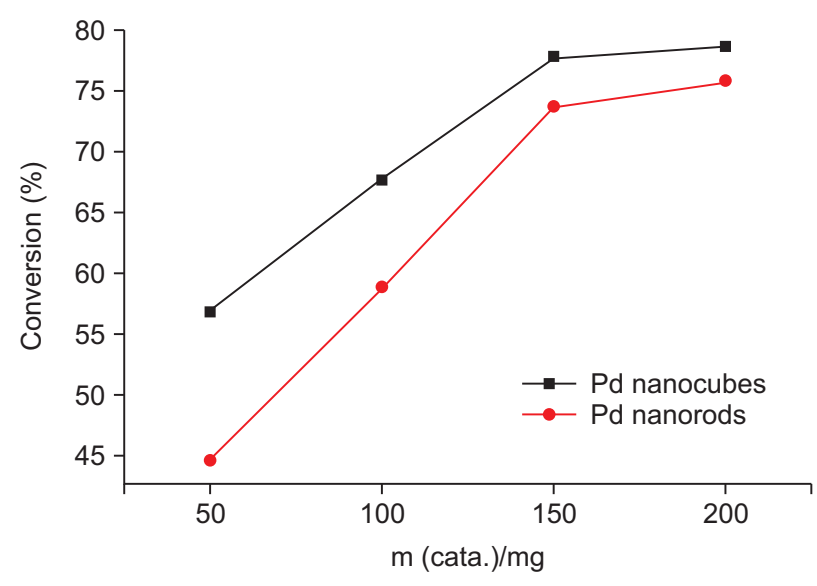

Fig. 5. Catalytic performance of Pd nanorods/nanocubes catalyst for Heck reaction of iodobenzene with acrylic acid methyl ester.

nanocubes groups were higher than Pd nanorods groups. We suspect that it is due to the different crystalline structure, which the Pd nanocubes are covered by $\{200\}$ plane while the Pd nanorods are covered by $\{111\}$ plane. It is caused by the difference of energy state while Pd nanocubes is enclosed by $\{200\}$ plane and nanorods is enclosed by $\{111\}$ plane. This is also consistent with other research result (Jin et al., 2011).

\section{CONCLUSIONS}

In conclusion, the single crystalline palladium nanocubes/ nanorods were prepared in the presence of PVP and $\mathrm{KBr}$ using the polyol method. The ratio of $\mathrm{KBr} / \mathrm{PVP}$ is the key factor to determine whether the product is cubes or rods. XRD, SADP and HRTEM shows that Pd nanocubes and nanorods were fcc structure with different surface, while Pd nanocubes is $\{200\}$ plane and nanorods is $\{111\}$ plane. The catalytic activity of these Pd nanocubes towards heck reaction of iodobenzene with acrylate or acrylic acid is found to be higher than that of Pd nanorods.

\section{CONFLICT OF INTEREST}

No potential conflict of interest relevant to this article was reported.

\section{ACKNOWLEDGMENTS}

Project Supported by Scientific and Technological Research Program of Chongqing Municipal Education Commission (Grant No. KJ1401330) and the cooperative project of academician workstation of Chongqing University of Science \& Technology (CKYS2014Y03\&CKYS2014Y08). 


\section{REFERENCES}

Baldauf M and Kolb D M (1996) Formic acid oxidation on ultrathin Pd films on Au(hkl) and Pt(hkl) electrodes. J. Phys. Chem. 100, 1137511381.

Burda C, Chen X B, Narayanan R, and El-Sayed M A (2005) Chemistry and properties of nanocrystals of different shapes. Chem. Rev. 105, 1025-1102.

Hoshi N, Kida K, Nakamura M, Nakada M, and Osada K (2006) Structural effects of electrochemical oxidation of formic acid on single crystal electrodes of palladium. J. Phys. Chem. B 110, 12480-12484.

Jin M S, Liu H Y, Zhang H, Xie Z X, Liu J Y, and Xia Y N (2011) Synthesis of $P d$ nanocrystals enclosed by $\{100\}$ facets and with sizes $<10 \mathrm{~nm}$ for application in CO oxidation. Nano Res. 4, 83-91.

Kim S W, Kim M, Lee W Y, and Hyeon T (2002) Fabrication of hollow palladium spheres and their successful application to the recyclable heterogeneous catalyst for suzuki coupling reactions. J. Am. Chem. Soc. 124, 7642-7643.

Kim S W, Park J, Jang Y, Chung Y, Hwang S, Hyeon T, and Kim Y W (2003) Synthesis of monodisperse palladium nanoparticles. Nano Lett. $\mathbf{3}$, 1289-1291.

Larsen R, Ha S, Zakzeski J, and Masel R I (2006) Unusually active palladium-based catalysts for the electrooxidation of formic acid. J. Power Sources 157, 78-84.

Li H Q, Sun G Q, Jiang Q, Zhu M Y, Sun S G, and Xin Q (2007) Preparation and characterization of $\mathrm{Pd} / \mathrm{C}$ catalyst obtained in NH3-mediated polyol process. J. Power Sources 172, 641-649.
Mazumder V and Sun S (2009) Oleylamine-mediated synthesis of Pd nanoparticles for catalytic formic acid oxidation. J. Am. Chem. Soc. 131, 4588-4589.

Nishihata Y, Mizuki J, Kao T, Tanaka H, Enishi M, Imura M, Kamoto T, and Hamada N (2002) Self-regeneration of a Pd-perovskite catalyst for automotive emissions control. Nature 418, 164-167.

Shen C M, Su Y K, Yang H T, Yang T Z, and Gao H J (2003) Synthesis and characterization of n-octadecayl mercaptan-protected palladium nanoparticles. Chem. Phys. Lett. 373, 39-45.

Wang R F, Liao S J, and Ji S (2008) High performance Pd-based catalysts for oxidation of formic acid. J. Power Sources 180, 205-208.

Xiao C W, Ding H, Shen C M, Yang T Z, Hui C, and Gao H J (2009) Shapecontrolled synthesis of palladium nanorods and their magnetic properties. J. Phys. Chem. C 113, 13466-13469.

Xiong Y, Chen J, Wiley B, Xia Y, Yin Y, and Li Z Y (2005) Size-dependence of surface plasmon resonance and oxidation for $\mathrm{Pd}$ nanocubes synthesized via a seed etching process. Nano Lett. 5, 1237-1242.

Xiong Y J, Cai H G, Wiley J B, Wang J G, Kim M J, and Xia Y N (2007) Synthesis and mechanistic study of palladium nanobars and nanorods. J. Am. Chem. Soc. 129, 3665-3675.

Xu C W, Cheng L Q, Shen P K, and Liu Y L (2007) Methanol and ethanol electrooxidation on $\mathrm{Pt}$ and $\mathrm{Pd}$ supported on carbon microspheres in alkaline media. Electrochem. Commun. 9, 997-1001. 